\title{
Cancer Cell Adhesion and Metastasis: Selectins, Integrins, and the Inhibitory Potential of Heparins
}

\author{
Gerd Bendas $^{1}$ and Lubor Borsig ${ }^{2}$ \\ ${ }^{1}$ Department of Pharmaceutical Chemistry, University of Bonn, 53121 Bonn, Germany \\ ${ }^{2}$ Institute of Physiology, University of Zürich and Zürich Center for Integrative Human Physiology, 8057 Zürich, Switzerland
}

Correspondence should be addressed to Lubor Borsig, lborsig@access.uzh.ch

Received 14 October 2011; Accepted 21 November 2011

Academic Editor: Martin Gotte

Copyright (C) 2012 G. Bendas and L. Borsig. This is an open access article distributed under the Creative Commons Attribution License, which permits unrestricted use, distribution, and reproduction in any medium, provided the original work is properly cited.

\begin{abstract}
Cell adhesion molecules play a significant role in cancer progression and metastasis. Cell-cell interactions of cancer cells with endothelium determine the metastatic spread. In addition, direct tumor cell interactions with platelets, leukocytes, and soluble components significantly contribute to cancer cell adhesion, extravasation, and the establishment of metastatic lesions. Clinical evidence indicates that heparin, commonly used for treatment of thromboembolic events in cancer patients, is beneficial for their survival. Preclinical studies confirm that heparin possesses antimetastatic activities that lead to attenuation of metastasis in various animal models. Heparin contains several biological activities that may affect several steps in metastatic cascade. Here we focus on the role of cellular adhesion receptors in the metastatic cascade and discuss evidence for heparin as an inhibitor of cell adhesion. While P- and L-selectin facilitation of cellular contacts during hematogenous metastasis is being accepted as a potential target of heparin, here we propose that heparin may also interfere with integrin activity and thereby affect cancer progression. This review summarizes recent findings about potential mechanisms of tumor cell interactions in the vasculature and antimetastatic activities of heparin.
\end{abstract}

\section{Introduction}

Metastasis is facilitated by cell-cell interactions between tumor cells and the endothelium in distant tissues. Tumor cells in circulation interact also with platelets and leukocytes that further contribute to tumor cell adhesion, extravasation, and the establishment of metastatic lesions. Hematogenous cancer metastasis is a multistep cascade encompassing process, starting with local invasion of tumor cells at primary tumors, survival in systemic circulation, extravasation in secondary sites, and ending with establishment of growing metastatic lesions. The metastatic capacity of tumor cells correlates with their ability to exit from the blood circulation, to colonize distant organs, and to grow in distant organs [1]. The organ-specific character of metastasis has been already observed by S. Paget more than a century ago, and the "seed and soil" hypothesis postulates specific interactions of tumor cells with the "friendly" environment of distant organs that enables the establishment of metastasis and subsequent growth [2]. While cell-cell interactions facilitating tumor cell adhesion in the vasculature of specific organs are essential steps in the metastatic cascade, inhibition of these interactions represent a therapeutically useful target for attenuation of metastasis (Figure 1). Two major cell adhesion molecule families, selectins and integrins, have been identified to participate in metastasis [3-6]. In addition to direct targeting of selectin and integrins, recent evidence suggests that heparin possesses selectin (P- and L-selectin) and also integrin inhibitory activity [7-9]. Heparin and low-molecularweight (LMW) heparin are commonly used anticoagulants for treatment of cancer-associated thromboembolism in cancer patients $[10,11]$. The exact mechanism of cellcell interactions involved in cancer progression remains to be fully elucidated; however, the potential of heparins to interfere with this process warrants further investigations. 


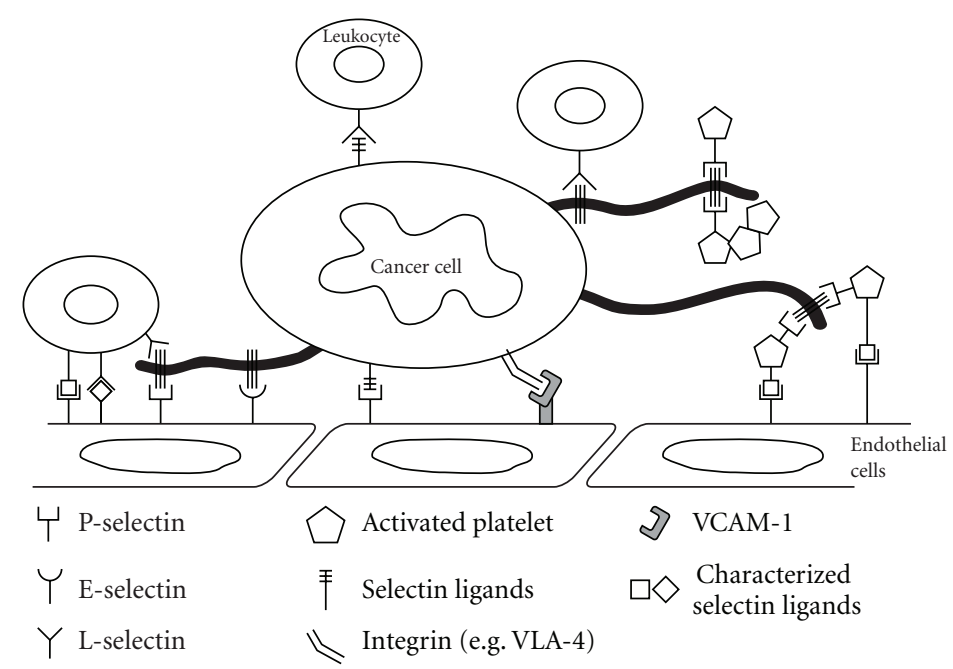

(a)

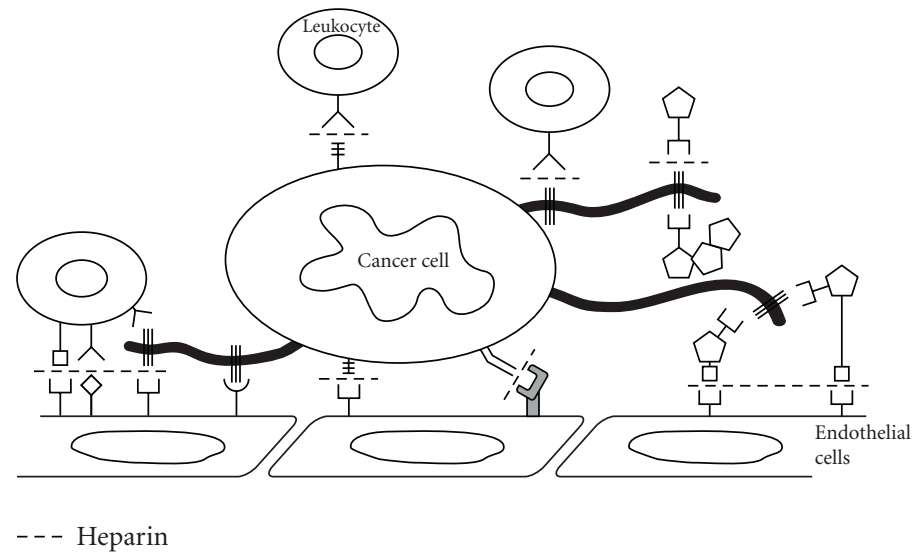

(b)

Figure 1: Selectins and integrins contribute to metastatic spread. (a) Schematic presentation of selectin- and integrin-mediated cancer cell interactions with several blood constituents (e.g., platelets, leukocytes, and endothelial cells) during hematogenous metastasis. (b) Heparin application in mouse models blocks both P- and L-selectin-mediated; and VLA4-mediated interactions of cancer cells within blood circulation thereby affecting metastasis.

\section{Cell Adhesion as a Determinant of Metastasis}

Within blood vessels, circulating tumor cells ultimately interact with the endothelium that might lead to tumor cell arrest and extravasation (Figure 1). Studies of tumor cellendothelial contact formations have been based on parallels to the leukocyte-endothelial cell interactions during inflammation. Although the mechanism of tumor cell adhesion certainly differs from leukocyte recruitment to inflammatory sites, the cell adhesion molecules involved in the contact formation with endothelium are potentially the same [3, $5,6]$. Indeed, there is accumulating evidence for the role of selectins and integrins in cancer progression of various cancer types, including colon and lung carcinomas and melanomas $[5,6]$. While selectin-mediated tumor cells arrest and adhesion contribute to metastasis, integrin-mediated interaction from both tumor cells and the surrounding environment further contribute to cancer progression.

\section{Selectins}

Selectins are vascular cell adhesion molecules involved in adhesive interactions of leukocytes and platelets and endothelium within the blood circulation. There are three members of the selectin family: P-, E-, and L-selectin. $\mathrm{P}$-selectin is present in the storage granules of platelets ( $\alpha$-granules) and endothelial cells (Weibel-Palade bodies), thus enabling rapid translocation on cell surfaces upon activation [12]. On the contrary, endothelial expression of Eselectin requires de novo transcription, leading to expression on activated endothelial cell surfaces several hours after stimulation [12]. L-selectin is constitutively expressed on cell surfaces of almost all leukocyte subpopulations. The physiological functions of selectins are well described in processes of inflammation, immune response, wound repair, and hemostasis [13]. The role of selectins in these processes has been elucidated in mouse deficient in individual selectins. 
Whereas L-selectin mediates fast rolling of leukocytes on endothelium, P- and E-selectins support rolling at lower velocities [13]. The initial steps in leukocyte tethering and rolling on endothelium are supported by rapid and reversible interactions of selectins with their carbohydrate ligands.

The majority of selectin ligands consist of distinct glycan structures carrying the terminal core tetrasaccharide structure sialyl Lewis ${ }^{\mathrm{x} / \mathrm{a}}\left(\mathrm{sLe}^{\mathrm{x}} / \mathrm{sLe}^{\mathrm{a}}\right)$ on a protein backbone [14, 15]. Selectins binds to various classes of molecules (mucins, sulfated glycolipids, glycosaminoglycans), and most of these molecules were shown to be functional selectin ligands in vivo [12]. Efficient selectin binding to carbohydrates usually requires a proper glycoprotein scaffold that presents several selectin ligands in clusters, thereby increasing the avidity of the interaction.

3.1. Selectins as Facilitators of Metastasis. Hematogenous metastasis is the common route for cancer spread of epithelial cancers-carcinomas. In the normal physiological state, epithelial cells line the lumen of hollow organs and are covered by mucins that are either cell surface attached or building soluble layers covering the epithelium. Mucins are high-molecular-weight molecules with a large proportion of O-linked glycans attached to a protein backbone. During malignant transformation cell surface glycans undergo dramatic changes [16]. The major alterations of glycans on tumor cells are associated with enhanced expression of $\mathrm{sLe}^{\mathrm{x}}$ or its isomer sLe $\mathrm{a}^{\mathrm{a}}$, Tn and sialyl-Tn antigen structures $[16,17]$. Enhanced expression of $s \mathrm{Le}^{\mathrm{x}}$ and sLe $\mathrm{a}^{\mathrm{a}}$ structures is frequently associated with progression and poor prognosis in various cancers including colon, gastric, lung, renal, and breast cancers, melanomas, and others (reviewed in [18]). Several laboratories have shown that at least one selectin (P-, $\mathrm{L}-$, or E-) is capable to bind to any human carcinoma tested (e.g., $[19,20])$, which emphasize the potential of selectins to mediate contacts with tumor cells within vasculature. The fact that, during the hematogenous phase of metastasis, selectin ligand-carrying tumor cells may encounter selectins, present on blood constituents (leukocytes, platelet, and endothelium) in the circulation, supported the notion of selectin involvement in metastatic progression $[3,5]$. This hypothesis has been evaluated by several laboratories, and recent findings using mouse models deficient in one or more selectins confirmed the involvement of $\mathrm{P}-, \mathrm{L}-$, and also Eselectin in metastasis [7, 21-24].

3.2. P-Selectin and Metastasis. The rapid expression of Pselectin on cell surfaces of endothelial cells and platelets upon activation makes P-selectin a likely candidate involved in the metastatic process. There is accumulating evidence that formation of platelet-tumor cell thrombi helps evading host responses, thereby contributing to metastasis [7, 25-27]. In the absence of platelet-tumor cell interactions, tumor cells are cleared by NK cells $[26,28]$. Minimal platelet-tumor cell microthrombi has been detected in the absence of P-selectin, leading to reduced tumor cell adhesion in the lungs of mice and subsequently attenuation of metastasis $[7,22,29]$. Furthermore, removal of cell surface mucins from tumor cells prior to intravenous injection resulted in reduction of metastasis [7]. Bone marrow reconstitution experiments in P-selectin-deficient mice have shown that endothelial Pselectin, in addition to platelet P-selectin, contributes to metastasis [29].

Patients with metastatic carcinoma cancer are at high risk for thromboembolic events, a finding initially described by Trousseau [30]. The association between mucinous carcinoma and Trousseau's syndrome led to the hypothesis that mucin might directly induce thrombi formation [31]. Intravenous injection of purified carcinoma mucin led to thrombi formation that was dependent both on the presence of P- and L-selectin. Recently, the molecular mechanism of mucin-induced initiation of tumor cell-platelet complexes has been described [32]. Microthrombi formation induced by carcinoma mucins was found to be dependent on Lselectin and PSGL-1 expression on neutrophils that induced cathepsin $\mathrm{G}$ release thereby triggering platelet activation and P-selectin expression.

3.3. L-Selectin and Metastasis. Participation of leukocytes in platelet-tumor cell emboli is well described, yet the role of leukocytes in the process of metastatic initiation remains under investigation. The establishment of a metastatic niche is based on the initial recruitment of bone marrow-derived cells to distant sites in organs where metastatic cells tend to seed $[33,34]$. In general, contribution of leukocytes to metastasis largely depends on spatial and temporal situation that is defined by the microenvironment and tumor cells [34-36]. Whether L-selectin mediates leukocyte recruitment to metastatic sites has been tested in L-selectin-deficient mice $[21,37]$. The absence of L-selectin led to significant attenuation of metastasis, indicating that L-selectin actively contributes to leukocyte recruitment and formation of a metastatic niche $[5,38]$. Reduced numbers of CD11bpositive leukocytes has been detected at early time points after tumor cell injection that correlated with reduced tumor cell survival in the lungs [37]. Recent evidence indicates that selectin-mediated interactions through cooption of inflammatory pathways contribute to formation of a permissive microenvironment for metastasis [38]. Tumor cell-mediated activation of the adjacent endothelium upon vascular arrest resulted in NF- $\kappa \mathrm{B}$ activation and expression of E-selectin, thereby contributing to metastasis.

3.4. E-Selectin and Metastasis. E-selectin has been investigated as a mediator of metastasis at sites where arrest of tumor cells in the microvasculature has been observed $[39,40]$. E-selectin expressed on activated endothelial cells has been detected during metastatic colonization of the liver $[39,41]$. Inhibition of E-selectin or downregulation of Eselectin expression resulted in attenuation of experimental liver metastasis. In contrast, transgenic overexpression of E-selectin in the liver redirected metastasis to this organ, thereby confirming the role of E-selectin in this process. Interestingly, experimental metastasis of human colon carcinoma cells lines was not affected by the absence of E-selectin [24]. However, spontaneous breast metastasis to the lung was 
reduced in E-selectin-deficient mice, indicating E-selectin involvement during lung metastasis [42]. Organ-specific differences may contribute to the colonization process and different requirements for selectin-mediated interactions may be dependent on the primary tumor and the metastatic organ.

While selectins were identified as potential facilitators of metastasis, they have not been explored as pharmacological targets for treating cancer progression.

\section{Biology of Integrins}

Integrins are large and complex transmembrane glycoproteins. The structure of integrins consists of two distinct chains, $\alpha$ - and $\beta$-subunit, which form a non-covalent heterodimer $[43,44]$. In mammals, $18 \alpha$-and $8 \beta$-integrins have been characterized that combine to form 24 unique canonical $\alpha / \beta$ receptors identified so far. Integrins mediate cell adhesion and directly bind components of the extracellular matrix (ECM), such as fibronectin, vitronectin, laminin, or collagen, thereby providing anchorage for cell motility and invasion. Since specific integrins can bind to different ligands and identical ligand can be shared by different integrins, this redundancy underscores the general importance of integrins in cell communication.

Integrins are mediators of a bidirectional signaling where intracellular signals induce alterations in the conformation, thus ligand-binding properties (inside-out signaling). Since integrins are linked to cytoskeletal structures (e.g., $\alpha$-actinin, talin, and vinculin) ligation of extracellular ligands can influence intracellular processes (outside-in signaling) through activation of kinases, GTPases of the Ras/Rho signaling pathways $[44,45]$. The convergence between the cytoskeleton and ECM components is mediated via a cluster formation of integrins and their downstream signaling molecules, focal adhesion kinase (FAK) or Src family kinases, which affect the cellular shape and migratory properties of cells [46]. In addition to the well-established role of integrins during migration and invasion, integrins also regulate cell proliferation, survival and angiogenesis, all processes actively investigated during cancer progression.

\section{Integrins during Cancer Metastasis}

The ubiquitous presence of integrins on tumor cells, blood components, vasculature, and stromal cells suggest that integrins might essentially contribute to different steps in the metastatic cascade. As many human tumors originate from epithelial cells, integrins expressed on epithelial cells are generally present also in tumor cells. Studies correlating integrin expression levels with the pathological outcomes, such as metastasis or patient survival, have identified several integrins that might be involved in cancer progression [6]. Tumor cell expression of $\alpha \mathrm{v} \beta 3, \alpha \mathrm{v} \beta 5, \alpha 5 \beta 1, \alpha 6 \beta 4$ correlated with metastatic progression in melanoma, breast carcinoma, prostate and pancreatic and lung cancer [6].

During hematogenous phase of metastasis tumor cell platelet interaction are mediated either by P-selectin (see above) or through platelet integrin $\alpha \operatorname{IIb} \beta 3$. Inhibition of $\alpha \operatorname{IIb} \beta 3$ integrin or P-selectin by function-blocking antibodies significantly reduced platelet-tumor cell interaction and tumor cell adhesion on activated endothelium [47-49]. Accordingly, attenuation of metastasis has been observed.

Tumor cell expression of $\alpha \mathrm{v} \beta 3$ integrin together with its capacity to bind several ECM components, including fibronectin, vitronectin, and osteopontin, has been regarded as critical factor for affecting the site of metastasis. In this respect, fibrinogen was identified as a bridging factor between $\alpha \operatorname{IIb} \beta 3$ integrins on platelet and $\alpha \mathrm{v} \beta 3$ on tumor cells $[50,51]$. This interaction facilitates tumor cell arrest in the vasculature and metastasis to various tissues, including bone marrow and lungs.

The contribution of integrins to angiogenesis and thereby tumor progression and metastasis has recently been reviewed [52]. Tumor-associated vessels express $\alpha \mathrm{v} \beta 3$ and $\alpha \mathrm{v} \beta 5$ integrins that were not detected in quiescent vasculature [53]. The binding of these vascular integrins to ECM components in the tumor microenvironment contributes to invasion and migration of endothelial cells. Therefore, targeting of $\alpha \mathrm{v} \beta 3$ and $\alpha \mathrm{v} \beta 5$ integrins with antibodies or peptide- (RGD-) derived structures has been investigated as a promising antiangiogenic approach.

The establishment of metastatic niche is dependent on the recruitment of bone marrow-derived cells [33, 34]. Homing of circulating progenitor cells to tumors was shown to require $\alpha 4 \beta 1$ integrins [54]. Expression of integrin $\alpha 4 \beta 1$ (VLA-4) on bone marrow-derived cells mediates binding to VCAM and cellular fibronectin, which are present at sites of endothelial remodeling.

Initial studies on the role of integrins during metastasis, specifically investigating primary endothelial contacts, were focused on melanoma metastasis [55-57]. Metastatic dissemination of murine melanoma B16F10 cells has been blocked by peptide displaying the RGD integrin-binding sequence of fibronectin [55]. The targeted integrin involved in the vascular arrest of melanoma cells has later been identified to be VLA-4 $[56,57]$. Experimental pulmonary metastasis of melanoma cell lines, B16 and A $375 \mathrm{M}$, was confirmed to be mediated by VLA-4. The specific VLA-4-mediated interaction with VCAM-1 on endothelium is required for melanoma cell adhesion and endothelial transmigration [58]. Recently, VLA-4 mediated melanoma adhesion to VCAM-1 on activated endothelium was shown to support extravasation under the shear flow also in the absence of selectin ligands [59], indicating the potential of VLA-4 to serve as an adhesion molecule during metastatic spread of cancer.

Besides the role of VLA- 4 in melanoma metastasis, $\beta 1$ integrins strongly contribute to metastasis of other tumor types, for example, lymphomas [60]. Silencing of $\beta 1$ integrins in the highly metastatic Esb murine T-lymphoma cell line, and thus the loss of VLA-4 and VLA- 6 binding, strongly reduced the metastatic dissemination to the lungs and spleen. Furthermore, a change in metastatic pattern with prevalence for skeletal muscle invasion has been observed [60]. To investigate the role of $\beta 1$ integrins during tumor growth and metastasis, a fibroblastoid cell line with disrupted $\beta 1$ integrin 
gene has been generated [61]. Overexpression of $\beta 1$ integrin significantly correlated with metastatic spread of these cells to the lung and liver, when compared with parental cells with disrupted $\beta 1$ integrin gene [61]. Taken together, integrins are becoming attractive therapeutic targets for therapeutic strategies focused not only on tumor development but also on metastasis.

Since heparins effectively block both P-, L-selectins and VLA-4 integrin-mediated tumor cell adhesion, heparin and heparin derivatives have been tested in a number of animal models for their potential to attenuate metastasis (reviewed in [9]).

\section{Heparin and Cancer: Clinical Evidence}

Heparin is commonly used for the prevention or treatment of venous thromboembolism in cancer patients. In addition to its antithrombotic activity, cancer patients treated with heparins showed an improved survival in a number of retrospective and prospective studies (reviewed in [62, 63]). A recent review on antithrombotic therapy using heparins concluded that, despite the heterogeneity of completed clinical studies, heparin treatment of cancer patients with better prognosis is beneficial for patients primarily due to prolonged survival [10]. Based on this conclusion, together with observations from animal models, heparin appears to directly affect cancer progression associated with metastatic spread.

\section{Heparin Attenuates Metastasis through Inhibition of Selectins}

Heparin is a complex mixture of natural glycosaminoglycans based on repeating disaccharide units containing glucosamine and glucuronic/iduronic acid residue with a high degree of sulfation [64]. Heparin and LMWH were tested in many different animal models for their potential to inhibit cancer progression primarily using experimental metastasis models (reviewed in $[65,66]$ ). Despite large variation in heparin preparations, doses applied, time of application, and different tumor models, attenuation of metastasis has been observed almost in all independent studies when heparin was applied around the time of tumor cell injection. Together with the limited effect on tumor growth $[67,68]$, these findings indicate that heparin affects processes when tumor cells are still in the blood circulation. Because of the very nature of heparin, several other biological activities in addition to the anticoagulant activity have been detected such as binding to cell adhesion molecules (P- and L-selectin, VLA-4 integrin), enzymes (heparanase), growth factors, and cytokines [6973]. Chemically modified heparins were prepared and tested for various biological activities [71]. Modified heparins containing mostly P-selectin inhibitory activity were shown to efficiently attenuate metastasis almost to the same levels as observed in P-selectin-deficient mice [71]. Since heparin injection in P-selectin-deficient mice resulted in no further attenuation of metastasis, these findings indicated that heparin affects metastasis by inhibition of P-selectin [7, 29,
71]. Interestingly, a single dose of heparin prior to tumor cell injection further attenuated metastasis in L-selectin-deficient mice, indicating that $\mathrm{L}$-selectin involvement in this process is subsequent to P-selectin [21]. Further evidence for sequential involvement of P- and L-selectin in metastasis was confirmed by observation that L-selectin contributes to metastasis first several hours after tumor cell injection [37]. Heparin injection 6-12 hours after the tumor cell challenge further reduced metastasis in $\mathrm{P}$-selectin-deficient mice confirming the potential of heparin to inhibit also L-selectin-mediated interactions. Taken together, these findings indicate that inhibition of P- and L-selectin attenuates metastasis and heparins appear to be efficient inhibitors of selectin mediated interactions in vivo.

\section{Heparin Binding and Inhibition of Integrin Functions}

Heparin as a potential inhibitor of integrin-mediated cellcell interaction has been evaluated only in few studies. The $\alpha \mathrm{M} \beta 2$ (Mac-1) integrin on hematopoietic progenitor cells was shown to mediate adhesion to stromal compartment through binding to heparin and heparan sulfate [74, 75]. Another study described the leukocyte integrin $\alpha \mathrm{X} \beta 2$ to bind sulfated heparin in a low micromolar range [76]. These studies suggest that heparin can interfere with leukocyte binding and recruitment to the endothelium.

Zhang et al. reported that platelet integrin $\alpha \mathrm{IIb} \beta 3$ can efficiently be blocked by heparin and non-anticoagulant heparin derivatives [77]. Platelet interaction with melanoma cells A375 or B16F10 was strongly reduced in vitro and metastasis in vivo. These findings indicate that heparin inhibition of $\alpha \operatorname{IIb} \beta 3$ integrins is additional to P-selectin inhibition of platelet binding (as mentioned above) with relevance for those tumors cells with low expression levels of P-selectin ligands.

Heparin inhibition of integrin-mediated melanoma adhesion to endothelium has been reported only recently [73]. Expression of integrin $\alpha 4 \beta 1$ (VLA-4) by B16F10 melanoma cells mediated their adhesion to endothelial cells through binding to VCAM-1. Heparin was shown to inhibit the VLA-4 mediated melanoma binding to VCAM-1 substrates under dynamic conditions. A follow-up study using human melanoma MV3 cells confirmed heparin binding to VLA4 with binding affinities in the low micromolar range [78]. Structural analysis of heparin indicated a size dependency of integrin binding, since short heparin fragment or the pentasaccharide (Fondaparinux) was not able to bind VLA-4 [8]. Further analysis revealed that also the sulfation density is critical for VLA-4 recognition [79].

Altogether, these studies indicate that heparin can affect important steps in the metastatic cascade by inhibition of integrins. The relevance of this contribution depends on tumor cell types carrying integrin and/or selectin ligands. Melanoma cells with their high expression levels of VLA-4 appear especially relevant for this consideration. However, the efficiency to inhibit metastasis in vivo remains to be analyzed. 


\section{Tumor Cell Seeding and the Establishment of Metastatic Niche}

Beside direct heparin inhibition of adhesion receptor functions, heparin can affect the activities of cellular proteoglycans related to cell adhesion. This possibility will shortly be introduced below with respect to potential antimetastatic approaches or novel targets.

Chemokines are chemotactic cytokines that induce direct migration of leukocytes to sites of inflammation or cancer progression [80]. Beside the well-described role of chemokines during inflammation, seeding of tumor cells to distant tissues was shown to be facilitated by chemokines.

There is compelling evidences that chemokine receptors, for example, CXCR4, mediate breast cancer metastasis [81]. Breast cancer cells expressing CXCR4 in circulation effectively enter the bone marrow niche due to enhanced expression of CXCL12 in this environment [82]. Targeted metastasis to the bone marrow or other sites with high expression of CXCL12 has been described in a number of cancers including breast, colon, and prostate [80]. Chemokines bind to glycosaminoglycans chains of proteoglycans presented on surfaces of epithelial and vascular endothelial cells or on extracellular matrix molecules. Cell migration is dependent on chemokine gradient presented by chemokines at specific sites [83]. Recently, syndecan-1 and syndecan-4 proteoglycans were shown to be required for chemokine- (CCL5-) induced hematoma migration and invasion [84]. Similarly, CCL2-induced human hepatoma cell migration and invasion has been blocked by anti-syndecan- 1 and -4 antibodies, but also when hepatoma cells were pretreated with heparitinases that remove glycosaminoglycans from cell surfaces [85]. Interestingly, a recent study reported on the ability of LMW heparin to bind SDF-1 (CXCL-12) in a sub-micromolar range [86]. Since proper chemokine presentation by endogenous proteoglycans is a prerequisite for successful metastasis, heparin treatment might "remove" chemokine as a decoy and thereby reduce tumor cell adhesion and recruitment to the metastatic sites. Chemokine binding to its chemokine receptor could directly activate integrin binding function by inducing the conformational change of the integrins [44]. Whether heparin binding to chemokines indeed attenuates metastasis remains to be explored.

Fibronectin, an ECM protein, possesses specific and partly overlapping binding sites for the integrin VLA-4, VLA5 , and heparin [87]. The anchorage of cells to heparinbinding domains of fibronectin or other ECM components is mainly linked to syndecans. Several studies point to the critical role of the syndecan extracellular domains in tumor cell adhesion and invasion behavior where syndecan- 4 acts as a "coreceptor" with integrin VLA-5, but not VLA-4 [43, 88]. Syndecan-4 binds to the extracellular matrix and is also connected to the actin cytoskeleton via interaction with structural and signaling proteins such as FAK, syndesmos, and paxillin. This represents both a mechanical and signaling link to cell surface integrin VLA-5 required for focal adhesion and stress fiber formation in cells adherent to fibronectin $[89,90]$. Thus, the potential heparin antimetastatic activity can be based on interference either directly with the integrin-fibronectin binding or indirectly via competing for the proteoglycan binding of syndecan-4 to fibronectin. However, a direct interference of heparin with this pathway in cell migration remains to be defined.

Integrins were also shown to bind cystein-rich protein 61-Cyr61 [91]. Cyr61 was first identified as a growth factorinducible immediate early gene and belongs to the CCN family of matricellular proteins (CCN1). Elevated levels of Cyr61 have been correlated with increased breast adenocarcinoma, endometrial tumors, pancreatic cancer, or glioma malignancy [92-95]. Cyr61 has been reported to mediate numerous cellular processes, such as cell adhesion, cell survival, proliferation, enhancement of growth factor-induced DNA synthesis, and angiogenesis [96]. These effects result, at least in part, through a direct binding of Cyr61 to the extracellular regions of the integrin $\alpha \mathrm{v} \beta 3$ thus activating these adhesion and signaling receptors [97]. However, Cyr61 has also a binding ability to $\alpha 2 \beta 1, \alpha 3 \beta 1, \alpha 5 \beta 1, \alpha 6 \beta 1$, and $\alpha \mathrm{M} \beta 2$ [91]. Cyr61 overexpression in gastric cancer cell line AGS was shown to increase peritoneal dissemination through increased $\alpha 2 \beta 1$ integrin activity [92]. In contrast, Cyr61 silencing in PC-3 and DU-145 prostate cancer cells strongly inhibited proliferation [98]. Though, Cyr61 also significantly enhanced TRAIL-induced apoptosis through interaction with integrins $\alpha \mathrm{v} \beta 3$ and $\alpha 6 \beta 4$ [98]. Therefore, inhibition of Cyr61 activity appears as a promising therapeutic approach to inhibit tumor cell growth, migration, and adhesion. The Cyr61 molecule has two discrete heparin binding sites which contribute to binding to cell surface on heparan sulfate proteoglycans on Syndecan-4 [91]. Consequently, heparin could indirectly influence integrin functions by depleting released Cyr61. Although, previous heparin treatment in a number of animal models might have affected also Cyr61 activity, further analysis is required to elucidate the relevance of this signaling pathway for antiadhesive approaches of heparin applications.

\section{Conclusions}

Accumulating evidence from several preclinical models confirms that tumor cell interactions through selectins and integrins actively contribute to the metastatic spread of tumor cells. However, the current cancer therapies are focused only on targeting of tumor cells while no specific therapy for inhibition of metastatic spread is available. Clinical findings suggest that heparin and LMW heparin possess anticancer activities leading to survival benefits for cancer patients in the early stage of the disease. Although the identification of underlying molecular mechanisms is still ongoing, several preclinical studies confirmed the dominant contribution of selectins to metastasis and their role as primary targets of heparin.

In addition, integrins were shown to contribute to cancer progression. Since heparin binds to particular integrins implicated in metastasis, targeting integrins opens additional ways for interference with metastatic progression in addition to inhibition of angiogenesis, cell migration, or establishment of the premetastatic niche. 
The elucidation of the orchestrated functions of selectin and integrins and possibly other adhesion molecules during metastatic cascade requires further studies. Clearly further clarification of heparin interactions with selectins and integrins is required, yet the abundant clinical experience with heparin and LMW heparin proposes its evaluation as a potential antimetastatic treatment.

\section{Acknowledgments}

This work was supported by grants from the Swiss National Science Foundation no. 31003A-133025 (to L. Borsig) and Deutsche Forschungsgemeinschaft (GRK 677 to G. Bendas).

\section{References}

[1] A. F. Chambers, A. C. Groom, and I. C. MacDonald, "Dissemination and growth of cancer cells in metastatic sites," Nature Reviews Cancer, vol. 2, no. 8, pp. 563-572, 2002.

[2] I. J. Fidler, "The pathogenesis of cancer metastasis: the "seed and soil" hypothesis revisited," Nature Reviews Cancer, vol. 3, no. 6, pp. 453-458, 2003.

[3] I. P. Witz, "The selectin-selectin ligand axis in tumor progression," Cancer and Metastasis Reviews, vol. 27, no. 1, pp. 19-30, 2008.

[4] K. A. Paschos, D. Canovas, and N. C. Bird, "The role of cell adhesion molecules in the progression of colorectal cancer and the development of liver metastasis," Cellular Signalling, vol. 21, no. 5, pp. 665-674, 2009.

[5] H. Läubli and L. Borsig, "Selectins promote tumor metastasis," Seminars in Cancer Biology, vol. 20, no. 3, pp. 169-177, 2010.

[6] J. S. Desgrosellier and D. A. Cheresh, "Integrins in cancer: biological implications and therapeutic opportunities," Nature Reviews Cancer, vol. 10, no. 1, pp. 9-22, 2010.

[7] L. Borsig, R. Wong, J. Feramisco, D. R. Nadeau, N. M. Varki, and A. Varki, "Heparin and cancer revisited: mechanistic connections involving platelets, $\mathrm{P}$-selectin, carcinoma mucins, and tumor metastasis," Proceedings of the National Academy of Sciences of the United States of America, vol. 98, no. 6, pp. 33523357, 2001.

[8] M. Schlesinger, A. Naggi, G. Torri et al., "Blocking of integrinmediated human MV3 melanoma cell binding by commercial and modified heparins," International Journal of Clinical Pharmacology and Therapeutics, vol. 48, no. 7, pp. 448-450, 2010.

[9] L. Borsig, "Selectins facilitate carcinoma metastasis and heparin can prevent them," News in Physiological Sciences, vol. 19, no. 1, pp. 16-21, 2004.

[10] A. K. Kakkar and F. MacBeth, "Antithrombotic therapy and survival in patients with malignant disease," British Journal of Cancer, vol. 102, no. 1, pp. S24-S29, 2010.

[11] L. R. Zacharski and J. T. Loynes, "Low-molecular-weight heparin in oncology," Anticancer Research, vol. 23, no. 3, pp. 2789 2793, 2003.

[12] G. S. Kansas, "Selectins and their ligands: current concepts and controversies," Blood, vol. 88, no. 9, pp. 3259-3287, 1996.

[13] K. Ley, C. Laudanna, M. I. Cybulsky, and S. Nourshargh, "Getting to the site of inflammation: the leukocyte adhesion cascade updated," Nature Reviews Immunology, vol. 7, no. 9, pp. 678-689, 2007.

[14] M. Sperandio, C. A. Gleissner, and K. Ley, "Glycosylation in immune cell trafficking," Immunological Reviews, vol. 230, no. 1, pp. 97-113, 2009.
[15] A. Varki, "Selectin ligands: will the real ones please stand up?" Journal of Clinical Investigation, vol. 99, no. 2, pp. 158-162, 1997.

[16] R. Kannagi, M. Izawa, T. Koike, K. Miyazaki, and N. Kimura, "Carbohydrate-mediated cell adhesion in cancer metastasis and angiogenesis," Cancer Science, vol. 95, no. 5, pp. 377-384, 2004.

[17] Y. J. Kim and A. Varki, "Perspectives on the significance of altered glycosylation of glycoproteins in cancer," Glycoconjugate Journal, vol. 14, no. 5, pp. 569-576, 1997.

[18] L. Borsig, J. L. Stevenson, and A. Varki, "Heparin in cancer: role of selectin interactions," in Cancer-Associated Thrombosis, A. A. Khorana and C. W. Francis, Eds., pp. 97-113, Informa Healthcare, New York, NY, USA, 2007.

[19] Y. J. Kim, L. Borsig, H. L. Han, N. M. Varki, and A. Varki, "Distinct selectin ligands on colon carcinoma mucins can mediate pathological interactions among platelets, leukocytes, and endothelium," American Journal of Pathology, vol. 155, no. 2, pp. 461-472, 1999.

[20] G. Mannori, P. Crottet, O. Cecconi et al., "Differential colon cancer cell adhesion to E-, P-, and L-selectin: role of mucintype glycoproteins," Cancer Research, vol. 55, no. 19, pp. 44254431, 1995.

[21] L. Borsig, R. Wong, R. O. Hynes, N. M. Varki, and A. Varki, "Synergistic effects of L- and P-selectin in facilitating tumor metastasis can involve non-mucin ligands and implicate leukocytes as enhancers of metastasis," Proceedings of the National Academy of Sciences of the United States of America, vol. 99, no. 4, pp. 2193-2198, 2002.

[22] Y. J. Kim, L. Borsig, N. M. Varki, and A. Varki, "P-selectin deficiency attenuates tumor growth and metastasis," Proceedings of the National Academy of Sciences of the United States of America, vol. 95, no. 16, pp. 9325-9330, 1998.

[23] S. Köhler, S. Ullrich, U. Richter, and U. Schumacher, "E/P-selectins and colon carcinoma metastasis: first in vivo evidence for their crucial role in a clinically relevant model of spontaneous metastasis formation in the lung," British Journal of Cancer, vol. 102, no. 3, pp. 602-609, 2010.

[24] H. Läubli and L. Borsig, "Selectins as mediators of lung metastasis," Cancer Microenvironment, vol. 3, no. 1, pp. 97$105,2010$.

[25] S. Karpatkin, E. Pearlstein, C. Ambrogio, and B. S. Coller, "Role of adhesive proteins in platelet tumor interaction in vitro and metastasis formation in vivo," Journal of Clinical Investigation, vol. 81, no. 4, pp. 1012-1019, 1988.

[26] B. Nieswandt, M. Hafner, B. Echtenacher, and D. N. Männel, "Lysis of tumor cells by natural killer cells in mice is impeded by platelets," Cancer Research, vol. 59, no. 6, pp. 1295-1300, 1999.

[27] K. V. Honn, D. G. Tang, and J. D. Crissman, "Platelets and cancer metastasis: a causal relationship?" Cancer and Metastasis Reviews, vol. 11, no. 3-4, pp. 325-351, 1992.

[28] M. M. Fuster, J. R. Brown, L. Wang, and J. D. Esko, "A disaccharide precursor of sialyl Lewis X inhibits metastatic potential of tumor cells," Cancer Research, vol. 63, no. 11, pp. 2775-2781, 2003.

[29] R. J. Ludwig, B. Boehme, M. Podda et al., "Endothelial Pselectin as a target of heparin action in experimental melanoma lung metastasis," Cancer Research, vol. 64, no. 8, pp. 27432750, 2004.

[30] A. Varki, “Trousseau's syndrome: multiple definitions and multiple mechanisms," Blood, vol. 110, no. 6, pp. 1723-1729, 2007. 
[31] M. Wahrenbrock, L. Borsig, D. Le, N. Varki, and A. Varki, "Selectin-mucin interactions as a probable molecular explanation for the association of Trousseau syndrome with mucinous adenocarcinomas," Journal of Clinical Investigation, vol. 112, no. 6, pp. 853-862, 2003.

[32] B. Shao, M. G. Wahrenbrock, L. Yao et al., "Carcinoma mucins trigger reciprocal activation of platelets and neutrophils in a murine model of Trousseau syndrome," Blood, vol. 118, no. 15, pp. 4015-4023, 2011.

[33] R. N. Kaplan, R. D. Riba, S. Zacharoulis et al., "VEGFR1positive haematopoietic bone marrow progenitors initiate the pre-metastatic niche," Nature, vol. 438, no. 7069, pp. 820-827, 2005.

[34] H. Peinado, S. Lavotshkin, and D. Lyden, "The secreted factors responsible for pre-metastatic niche formation: old sayings and new thoughts," Seminars in Cancer Biology, vol. 21, no. 2, pp. 139-146, 2011.

[35] A. Mantovani, P. Allavena, A. Sica, and F. Balkwill, "Cancerrelated inflammation," Nature, vol. 454, no. 7203, pp. 436-444, 2008.

[36] L. M. Coussens and Z. Werb, "Inflammation and cancer," Nature, vol. 420, no. 6917, pp. 860-867, 2002.

[37] H. Läubli, J. L. Stevenson, A. Varki, N. M. Varki, and L. Borsig, "L-selectin facilitation of metastasis involves temporal induction of Fut7-dependent ligands at sites of tumor cell arrest," Cancer Research, vol. 66, no. 3, pp. 1536-1542, 2006.

[38] H. Läubli, K. S. Spanaus, and L. Borsig, "Selectin-mediated activation of endothelial cells induces expression of CCL5 and promotes metastasis through recruitment of monocytes," Blood, vol. 114, no. 20, pp. 4583-4591, 2009.

[39] A. M. Khatib, M. Kontogiannea, L. Fallavollita, B. Jamison, S. Meterissian, and P. Brodt, "Rapid induction of cytokine and Eselectin expression in the liver in response to metastatic tumor cells," Cancer Research, vol. 59, no. 6, pp. 1356-1361, 1999.

[40] S. Gout, P. L. Tremblay, and J. Huot, "Selectins and selectin ligands in extravasation of cancer cells and organ selectivity of metastasis," Clinical and Experimental Metastasis, vol. 25, no. 4, pp. 335-344, 2008.

[41] P. Brodt, L. Fallavollita, R. S. Bresalier, S. Meterissian, C. R. Norton, and B. A. Wolitzky, "Liver endothelial E-selectin mediates carcinoma cell adhesion and promotes liver metastasis," International Journal of Cancer, vol. 71, no. 4, pp. 612-619, 1997.

[42] S. Hiratsuka, S. Goel, W. S. Kamoun et al., "Endothelial focal adhesion kinase mediates cancer cell homing to discrete regions of the lungs via E-selectin up-regulation," Proceedings of the National Academy of Sciences of the United States of America, vol. 108, no. 9, pp. 3725-3730, 2011.

[43] M. R. Morgan, M. J. Humphries, and M. D. Bass, "Synergistic control of cell adhesion by integrins and syndecans," Nature Reviews Molecular Cell Biology, vol. 8, no. 12, pp. 957-969, 2007.

[44] R. O. Hynes, "Integrins: bidirectional, allosteric signaling machines," Cell, vol. 110, no. 6, pp. 673-687, 2002.

[45] T. L. Lau, C. Kim, M. H. Ginsberg, and T. S. Ulmer, "The structure of the integrin alphalIbbeta3 transmembrane complex explains integrin transmembrane signalling," EMBO Journal, vol. 28, no. 9, pp. 1351-1361, 2009.

[46] S. Huveneers and E. H. J. Danen, "Adhesion signalingcrosstalk between integrins, Src and Rho," Journal of Cell Science, vol. 122, no. 8, pp. 1059-1069, 2009.

[47] R. Dardik, N. Savion, Y. Kaufmann, and D. Varon, "Thrombin promotes platelet-mediated melanoma cell adhesion to endothelial cells under flow conditions: role of platelet glycoproteins P-selectin and GPIIb-IIIA," British Journal of Cancer, vol. 77, no. 12, pp. 2069-2075, 1998.

[48] M. L. Nierodzik, A. Klepfish, and S. Karpatkin, "Role of platelets, thrombin, integrin IIb-IIIa, fibronectin and von Willebrand factor on tumor adhesion in vitro and metastasis in vivo," Thrombosis and Haemostasis, vol. 74, no. 1, pp. 282290, 1995.

[49] H. Boukerche, O. Berthier-Vergnes, E. Tabone, J. F. Dore, L. L. K. Leung, and J. L. McGregor, "Platelet-melanoma cell interaction is mediated by the glycoprotein IIb-IIIa complex," Blood, vol. 74, no. 2, pp. 658-663, 1989.

[50] B. Felding-Habermann, R. Habermann, E. Saldívar, and Z. M. Ruggeri, "Role of $\beta 3$ integrins in melanoma cell adhesion to activated platelets under flow," Journal of Biological Chemistry, vol. 271, no. 10, pp. 5892-5900, 1996.

[51] B. Felding-Habermann, T. E. O'Toole, J. W. Smith et al., "Integrin activation controls metastasis in human breast cancer," Proceedings of the National Academy of Sciences of the United States of America, vol. 98, no. 4, pp. 1853-1858, 2001.

[52] C. J. Avraamides, B. Garmy-Susini, and J. A. Varner, "Integrins in angiogenesis and lymphangiogenesis," Nature Reviews Cancer, vol. 8, no. 8, pp. 604-617, 2008.

[53] P. C. Brooks, R. A. F. Clark, and D. A. Cheresh, "Requirement of vascular integrin $\alpha(\mathrm{v}) \beta 3$ for angiogenesis," Science, vol. 264, no. 5158, pp. 569-571, 1994.

[54] H. Jin, A. Aiyer, J. Su et al., "A homing mechanism for bone marrow-derived progenitor cell recruitment to the neovasculature," Journal of Clinical Investigation, vol. 116, no. 3, pp. 652-662, 2006.

[55] M. J. Humphries, K. M. Yamada, and K. Olden, "Investigation of the biological effects of anti-cell adhesive synthetic peptides that inhibit experimental metastasis of B16-F10 murine melanoma cells," Journal of Clinical Investigation, vol. 81, no. 3, pp. 782-790, 1988.

[56] H. Okahara, H. Yagita, K. Miyake, and K. Okumura, "Involvement of very late activation antigen 4 (VLA-4) and vascular cell adhesion molecule 1 (VCAM-1) in tumor necrosis factor $\alpha$ enhancement of experimental metastasis," Cancer Research, vol. 54, no. 12, pp. 3233-3236, 1994.

[57] A. Garofalo, R. G. S. Chirivi, C. Foglieni et al., "Involvement of the very late antigen 4 integrin on melanoma in interleukin 1 -augmented experimental metastases," Cancer Research, vol. 55, no. 2, pp. 414-419, 1995.

[58] M. Klemke, T. Weschenfelder, M. H. Konstandin, and Y. Samstag, "High affinity interaction of integrin $\alpha 4 \beta 1$ (VLA4) and vascular cell adhesion molecule 1 (VCAM-1) enhances migration of human melanoma cells across activated endothelial cell layers," Journal of Cellular Physiology, vol. 212, no. 2, pp. 368-374, 2007.

[59] S. Liang and C. Dong, "Integrin VLA-4 enhances sialylLewisx/a-negative melanoma adhesion to and extravasation through the endothelium under low flow conditions," American Journal of Physiology, vol. 295, no. 3, pp. C701-C707, 2008.

[60] P. J. M. Strocken, E. A. M. Van Rijthoven, M. A. Van Der Valk, and E. Roos, "Targeted disruption of the $\beta 1$ integrin gene in a lymphoma cell line greatly reduces metastatic capacity," Cancer Research, vol. 58, no. 7, pp. 1569-1577, 1998.

[61] C. Brakebusch, K. Wennerberg, H. W. Krell et al., " $\beta 1$ integrin promotes but is not essential for metastasis of ras-myc transformed fibroblasts," Oncogene, vol. 18, no. 26, pp. 3852-3861, 1999.

[62] N. M. Kuderer, A. A. Khorana, G. H. Lyman, and C. W. Francis, "A meta-analysis and systematic review of the efficacy 
and safety of anticoagulants as cancer treatment: impact on survival and bleeding complications," Cancer, vol. 110, no. 5, pp. 1149-1161, 2007.

[63] V. Tagalakis, M. Blostein, C. Robinson-Cohen, and S. R. Kahn, "The effect of anticoagulants on cancer risk and survival: systematic review," Cancer Treatment Reviews, vol. 33, no. 4, pp. 358-368, 2007.

[64] J. D. Esko and U. Lindahl, "Molecular diversity of heparan sulfate," Journal of Clinical Investigation, vol. 108, no. 2, pp. 169-173, 2001.

[65] L. Borsig, "Antimetastatic activities of heparins and modified heparins. Experimental evidence," Thrombosis research, vol. 125, supplement, pp. S66-S71, 2010.

[66] T. M. H. Niers, C. P. W. Klerk, M. DiNisio et al., "Mechanisms of heparin induced anti-cancer activity in experimental cancer models," Critical Reviews in Oncology/Hematology, vol. 61, no. 3, pp. 195-207, 2007.

[67] T. Sciumbata, P. Caretto, P. Pirovano et al., "Treatment with modified heparins inhibits experimental metastasis formation and leads, in some animals, to long-term survival," Invasion and Metastasis, vol. 16, no. 3, pp. 132-143, 1996.

[68] M. Kragh, L. Binderup, P. J. Vig Hjarnaa, E. Bramm, K. B. Johansen, and C. Frimundt Petersen, "Non-anti-coagulant heparin inhibits metastasis but not primary tumor growth," Oncology Reports, vol. 14, no. 1, pp. 99-104, 2005.

[69] B. Casu, A. Naggi, and G. Torri, "Heparin-derived heparan sulfate mimics to modulate heparan sulfate-protein interaction in inflammation and cancer," Matrix Biology, vol. 29, no. 6, pp. 442-452, 2010.

[70] A. Koenig, K. Norgard-Sumnicht, R. Linhardt, and A. Varki, "Differential interactions of heparin and heparan sulfate glycosaminoglycans with the selectins: implications for the use of unfractionated and low molecular weight heparins as therapeutic agents," Journal of Clinical Investigation, vol. 101, no. 4, pp. 877-889, 1998.

[71] N. Hostettler, A. Naggi, G. Torri et al., "P-selectin- and heparanase-dependent antimetastatic activity of non-anticoagulant heparins," FASEB Journal, vol. 21, no. 13, pp. 3562 3572, 2007.

[72] A. Naggi, B. Casu, M. Perez et al., "Modulation of the heparanase-inhibiting activity of heparin through selective desulfation, graded N-acetylation, and glycol splitting," Journal of Biological Chemistry, vol. 280, no. 13, pp. 12103-12113, 2005.

[73] J. Fritzsche, D. Simonis, and G. Bendas, "Melanoma cell adhesion can be blocked by heparin in vitro: suggestion of VLA-4 as a novel target for antimetastatic approaches," Thrombosis and Haemostasis, vol. 100, no. 6, pp. 1166-1175, 2008.

[74] D. R. Coombe, S. M. Watt, and C. R. Parish, "Mac-1 (CD11b/CD18) and CD45 mediate the adhesion of hematopoietic progenitor cells to stromal cell elements via recognition of stromal heparan sulfate," Blood, vol. 84, no. 3, pp. 739-752, 1994.

[75] M. S. Diamond, R. Alon, C. A. Parkos, M. T. Quinn, and T. A. Springer, "Heparin is an adhesive ligand for the leukocyte integrin Mac-1 (CD11b/CD18)," Journal of Cell Biology, vol. 130, no. 6, pp. 1473-1482, 1995.

[76] T. Vorup-Jensen, L. Chi, L. C. Gjelstrup et al., "Binding between the integrin $\alpha \mathrm{X} \beta 2$ (CD11c/CD18) and heparin," Journal of Biological Chemistry, vol. 282, no. 42, pp. 30869-30877, 2007.

[77] C. Zhang, Y. Liu, Y. Gao et al., "Modified heparins inhibit integrin $\alpha \mathrm{IIb} \beta 3$ mediated adhesion of melanoma cells to platelets in vitro and in vivo," International Journal of Cancer, vol. 125, no. 9, pp. 2058-2065, 2009.

[78] M. Schlesinger, D. Simonis, P. Schmitz, J. Fritzsche, and G. Bendas, "Binding between heparin and the integrin VLA-4," Thrombosis and Haemostasis, vol. 102, no. 5, pp. 816-822, 2009.

[79] M. Schlesinger, P. Schmitz, R. Zeisig et al., "The inhibition of the integrin VLA-4 in MV3 melanoma cell binding by nonanticoagulant heparin derivatives," Thrombosis Research. In press.

[80] F. Balkwill, "Cancer and the chemokine network," Nature Reviews Cancer, vol. 4, no. 7, pp. 540-550, 2004.

[81] J. R. Harvey, P. Mellor, H. Eldaly, T. W. J. Lennard, J. A. Kirby, and S. Ali, "Inhibition of CXCR4-mediated breast cancer metastasis: a potential role for heparinoids?" Clinical Cancer Research, vol. 13, no. 5, pp. 1562-1570, 2007.

[82] J. A. Burger and T. J. Kipps, "CXCR4: a key receptor in the crosstalk between tumor cells and their microenvironment," Blood, vol. 107, no. 5, pp. 1761-1767, 2006.

[83] A. J. Hoogewerf, G. S. V. Kuschert, A. E. I. Proudfoot et al., "Glycosaminoglycans mediate cell surface oligomerization of chemokines," Biochemistry, vol. 36, no. 44, pp. 13570-13578, 1997.

[84] F. Charni, V. Friand, O. Haddad et al., "Syndecan-1 and syndecan-4 are involved in RANTES/CCL5-induced migration and invasion of human hepatoma cells," Biochimica et Biophysica Acta, vol. 1790, no. 10, pp. 1314-1326, 2009.

[85] M. Dagouassat, N. Suffee, H. Hlawaty et al., "Monocyte chemoattractant protein-1 (MCP-1)/CCL2 secreted by hepatic myofibroblasts promotes migration and invasion of human hepatoma cells," International Journal of Cancer, vol. 126, no. 5, pp. 1095-1108, 2010.

[86] H. Zhou, S. Roy, E. Cochran et al., "M402, a novel Heparan sulfate mimetic, targets multiple pathways implicated in tumor progression and metastasis," PLoS One, vol. 6, no. 6, article e21106, 2011.

[87] A. Sharma, J. A. Askari, M. J. Humphries, E. Yvonne Jones, and D. I. Stuart, "Crystal structure of a heparin- and integrinbinding segment of human fibronectin," EMBO Journal, vol. 18, no. 6, pp. 1468-1479, 1999.

[88] Z. Mostafavi-Pour, J. A. Askari, S. J. Parkinson, P. J. Parker, T. T. C. Ng, and M. J. Humphries, "Integrin-specific signaling pathways controlling focal adhesion formation and cell migration," Journal of Cell Biology, vol. 161, no. 1, pp. 155-167, 2003.

[89] L. Bloom, K. C. Ingham, and R. O. Hynes, "Fibronectin regulates assembly of actin filaments and focal contacts in cultured cells via the heparin-binding site in repeat III13," Molecular Biology of the Cell, vol. 10, no. 5, pp. 1521-1536, 1999.

[90] A. Woods and J. R. Couchman, "Syndecan 4 heparan sulfate proteoglycan is a selectively enriched and widespread focal adhesion component," Molecular Biology of the Cell, vol. 5, no. 2, pp. 183-192, 1994.

[91] C. T. Walsh, J. Radeff-Huang, R. Matteo et al., "Thrombin receptor and RhoA mediate cell proliferation through integrins and cysteine-rich protein 61," FASEB Journal, vol. 22, no. 11, pp. 4011-4021, 2008.

[92] M. T. Lin, C. C. Chang, B. R. Lin et al., "Elevated expression of Cyr61 enhances peritoneal dissemination of gastric cancer cells through integrin $\alpha 2 \beta 1$," Journal of Biological Chemistry, vol. 282, no. 47, pp. 34594-34604, 2007.

[93] M. S. Tsai, D. F. Bogart, J. M. Castañeda, P. Li, and R. Lupu, "Cyr61 promotes breast tumorigenesis and cancer progression,” Oncogene, vol. 21, no. 53, pp. 8178-8185, 2002. 
[94] S. E. Holloway, A. W. Beck, L. Girard et al., "Increased expression of Cyr61 (CCN1) identified in peritoneal metastases from human pancreatic cancer," Journal of the American College of Surgeons, vol. 200, no. 3, pp. 371-377, 2005.

[95] H. Watari, Y. Xiong, M. K. Hassan, and N. Sakuragi, "Cyr61, a member of ccn (connective tissue growth factor/cysteine-rich 61/nephroblastoma overexpressed) family, predicts survival of patients with endometrial cancer of endometrioid subtype," Gynecologic Oncology, vol. 112, no. 1, pp. 229-234, 2009.

[96] A. M. Babic, M. L. Kireeva, T. V. Kolesnikova, and L. F. Lau, "CYR61, a product of a growth factor-inducible immediate early gene, promotes angiogenesis and tumor growth," Proceedings of the National Academy of Sciences of the United States of America, vol. 95, no. 11, pp. 6355-6360, 1998.

[97] A. M. Babic, C. C. Chen, and L. F. Lau, "Fisp12/mouse connective tissue growth factor mediates endothelial cell adhesion and migration through integrin $\alpha(\mathrm{v}) \beta 3$, promotes endothelial cell survival, and induces angiogenesis in vivo," Molecular and Cellular Biology, vol. 19, no. 4, pp. 2958-2966, 1999.

[98] C. A. Franzen, C. C. Chen, V. Todorović, V. Juric, R. I. Monzon, and L. F. Lau, "Matrix protein CCN1 is critical for prostate carcinoma cell proliferation and TRAIL-induced apoptosis," Molecular Cancer Research, vol. 7, no. 7, pp. 1045-1055, 2009. 

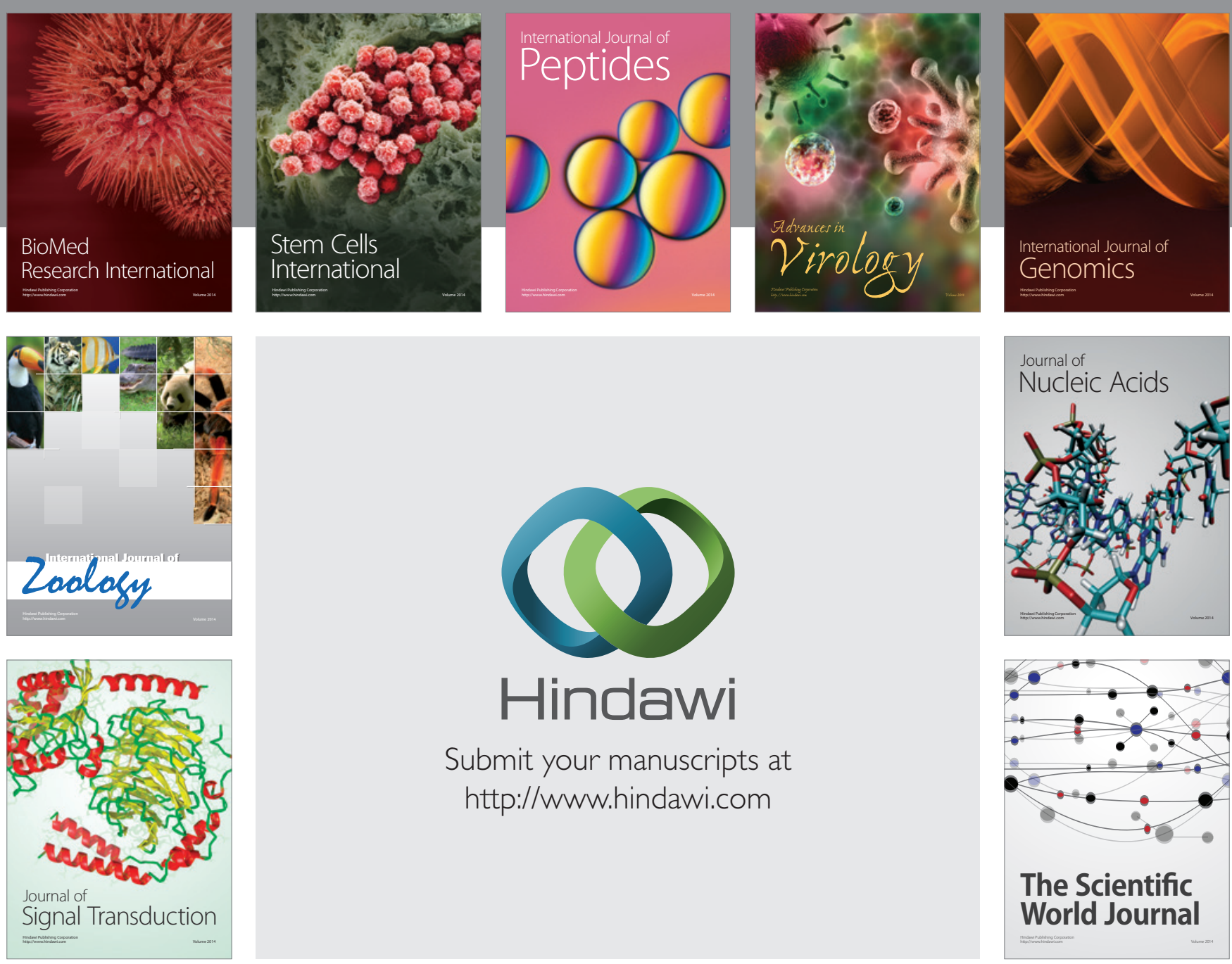

Submit your manuscripts at

http://www.hindawi.com
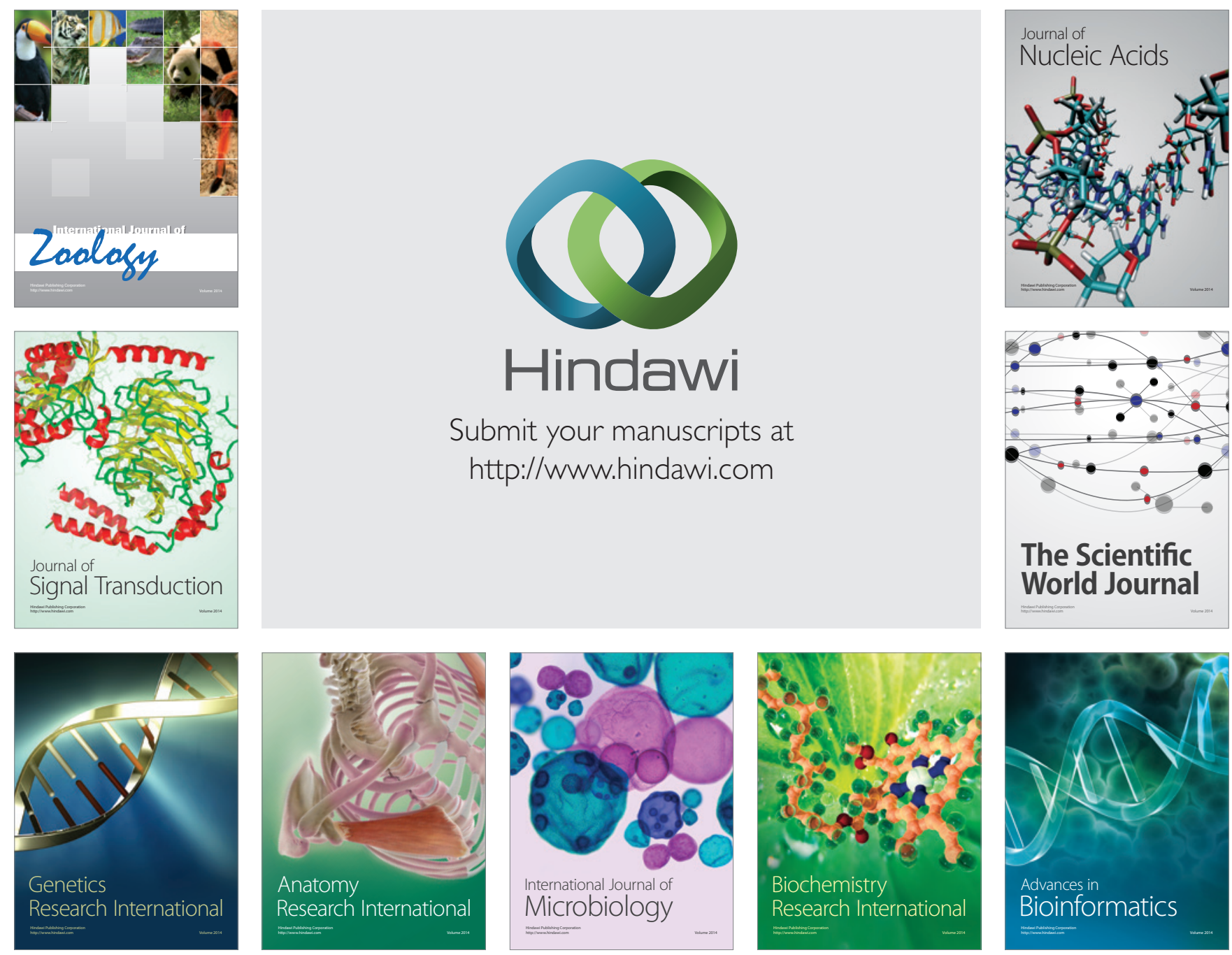

The Scientific World Journal
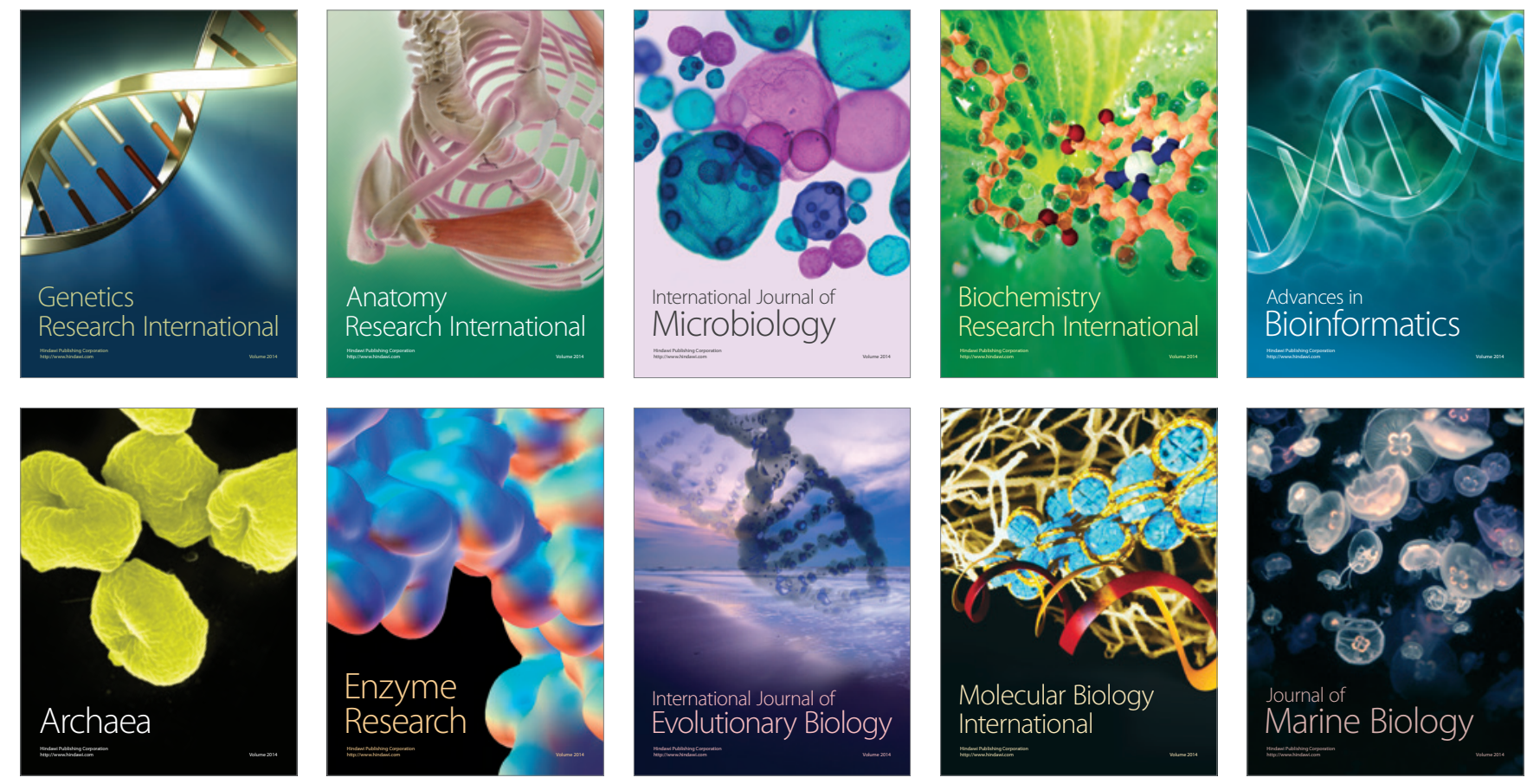